\title{
Measurements of Higgs boson properties using a combination of different Higgs decay channels
}

\author{
Nicolas Morange*, on behalf of the ATLAS Collaboration \\ LAL, Université Paris-Sud, CNRS/IN2P3, Université Paris-Saclay, Orsay, France \\ E-mail: nicolas.morange@cern.ch
}

\begin{abstract}
Six years after the first observation of the Higgs boson, the exploration of the Higgs sector of the Standard Model lagrangian remains at full swing. Analysing up to $80 \mathrm{fb}^{-1}$ of $13 \mathrm{TeV}$ collision data recorded during the Run-2 of the LHC, the ATLAS collaboration has produced a number of measurements on the Higgs boson in all its main production modes and in many decay channels. These analyses are combined to measure differential distributions or extract information on the couplings of the Higgs boson. This in turns allows to test the Standard Model predictions in the Higgs boson sector in terms of distributions and couplings, and to set constraints on different scenarios of physics beyond the Standard Model.
\end{abstract}

The 39th International Conference on High Energy Physics (ICHEP2018)

4-11 July, 2018

Seoul, Korea

${ }^{*}$ Speaker. 


\section{Introduction}

The phenomenology of the Higgs boson is particularly rich at the LHC. It is produced through four main modes that are experimentally accessible, and a number of decay channels can be probed. That gives rise to many experimental signatures, which are searched for by the Higgs analyses of the ATLAS collaboration using up to $80 \mathrm{fb}^{-1}$ of $13 \mathrm{TeV} p p$ collision data collected during the Run-2 of the LHC.

This contribution first presents a combination of the measurements in the $H \rightarrow Z Z^{*} \rightarrow 4 \ell$ and $H \rightarrow \gamma \gamma$ channels, of several differential distributions of the Higgs boson. The results of the combination of all the main Higgs analysis channels, interpreted in terms of Higgs boson signal strengths and couplings, are then discussed. As the channels included in the combination cover all the main production and decay modes, their combination provides stringent tests of the validity of the Standard Model (SM), and allows to set limits on beyond-the-standard-model (BSM) physics scenarios.

\section{Differential distributions}

The precise reconstruction of the final state achieved in the $H \rightarrow \gamma \gamma$ and $H \rightarrow Z Z^{*} \rightarrow 4 \ell$ channels makes it possible to measure with good accuracy differential distributions of the Higgs boson kinematics and of related event variables. The individual measurements made using $36.1 \mathrm{fb}^{-1}$ of data $[1,2]$ have been combined [3]. The total cross-section is measured to be $57.0_{-5.9}^{+6.0}$ (stat. $)_{-3.3}^{+4.0}$ (syst.) pb. The distributions of $p_{\mathrm{T}}^{H},\left|y^{H}\right|, N_{\text {jets }}$ and $p_{\mathrm{T}}^{j 1}$ are measured and compared to state-of-the-art calculations. Figure 1 shows the $p_{\mathrm{T}}^{H}$ and $N_{\text {jets }}$ distributions. All bins are dominated by statistical uncertainties at the 20-30\% level. The two channels are in good agreement with each other, and the SM predictions are compatible with the measurements, with discrepancies always below $3 \sigma$ in any bin.

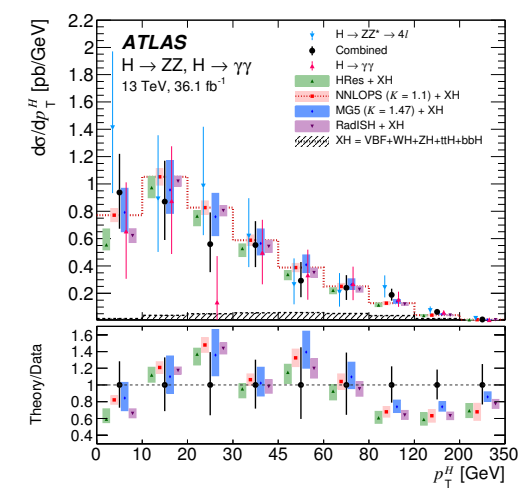

(a) Higgs boson transverse momentum

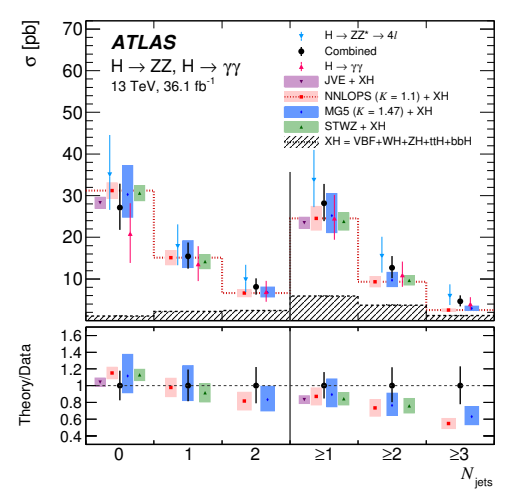

(b) Number of jets with pT $>30 \mathrm{GeV}$

Figure 1: Differential cross sections in the full phase space measured with the $H \rightarrow \gamma \gamma$ (red upward triangle) and $H \rightarrow Z Z^{*} \rightarrow 4 \ell$ (blue downward triangle) decay channels, as well as the combined measurement (black circle). Different SM predictions are overlaid as colored points, with the colored boxes indicating their uncertainties. 


\section{Higgs boson couplings}

Seven main analyses are combined together [4] to probe the Higgs boson couplings. The $H \rightarrow \gamma \gamma$ [5], $H \rightarrow Z Z^{*} \rightarrow 4 \ell[6]$ and $H \rightarrow \mu \mu$ [10] analyses use $80 \mathrm{fb}^{-1}$ of $13 \mathrm{TeV}$ data. The $V H(b \bar{b})$ [9], $t \bar{t} H$ [11] and $H \rightarrow \tau \tau$ [8] analyses use the data collected in 2015 and 2016, which amounts to $36.1 \mathrm{fb}^{-1}$.

The most basic measurement of the combination is that of a global signal strength, defined as a global scaling of the SM expected Higgs boson yields in all channels by a single value. It is found to be $\mu=1.13 \pm 0.05$ (stat.) \pm 0.05 (exp. $)_{-0.04}^{+0.05}$ (sig.th.) \pm 0.03 (bkg.th.), compatible with the SM at $13 \%$ level. Given the significant impact of the signal theory uncertainties in this result, it becomes more relevant to measure cross-sections instead.

The cross-sections for the Higgs production modes are then measured, assuming the SM branching ratios. A single-experiment observation of the VBF production mode is achieved, leaving $V H$ as the only unobserved mode. In the dominant $\mathrm{ggF}$ mode, the measured uncertainty is not far from the precision of the theory prediction. The correlations between the production modes are rather low, the largest being $14 \%$ between the ggF and VBF modes. More model-independent results are obtained when using the process $g g \rightarrow Z Z^{*} \rightarrow 4 \ell$ as a reference and measuring all production modes and branching ratios relative to it. All the measured values are compatible with the SM prediction.

The global combination is also interpreted in terms of effective coupling strengths modifiers as defined in the so-called $\kappa$-framework:

$$
\sigma_{i} \cdot \mathrm{B}_{f}=\kappa_{i}^{2} \sigma_{i}^{\mathrm{SM}} \frac{\kappa_{f}^{2} \Gamma_{f}^{\mathrm{SM}}}{\kappa_{H}^{2} \Gamma_{H}^{\mathrm{SM}}}
$$

Different parameterisations are used to probe the Higgs sector of the Lagriangian in various ways. Figure 2a shows the result of the fit to effective photon and gluon couplings, that probes for non-SM contributions in the loops of the $H \gamma \gamma$ and $H g g$ vertices: the compatibility with the SM is $70.5 \%$. In contrast, Figure $2 \mathrm{~b}$ presents the result of a consistency test of the SM, which assumes the SM structure of the loops and no BSM decays for the Higgs boson. In another parameterisation, which adds the effective photon and gluon couplings and allows for BSM decays, a limit on the branching ratio to BSM decays $B_{\mathrm{BSM}}<0.26$ can be set under fairly generic assumptions. Finally, more model-independent results are obtained by measuring ratios of coupling modifiers. Results show there again an excellent compatibility with the SM predictions.

The measurements are also interpreted in benchmark scenarios of BSM physics, namely the Two Higgs Doublet Models (2HDM) and the hMSSM, a simplified version of the Minimal Supersymmetric Standard Model. Exclusion limit are presented in the $(\cos (\beta-\alpha), \tan \beta)$ planes of the four types of 2HDM. The data are consistent with the alignment limit (i.e SM), with the exception of a narrow petal, where fermion couplings have the same magnitude but an opposite sign compared to the SM. In the hMSSM, CP-odd Higgs boson masses below $520 \mathrm{GeV}$ are excluded, and the data are compatible with the decoupling limit. 


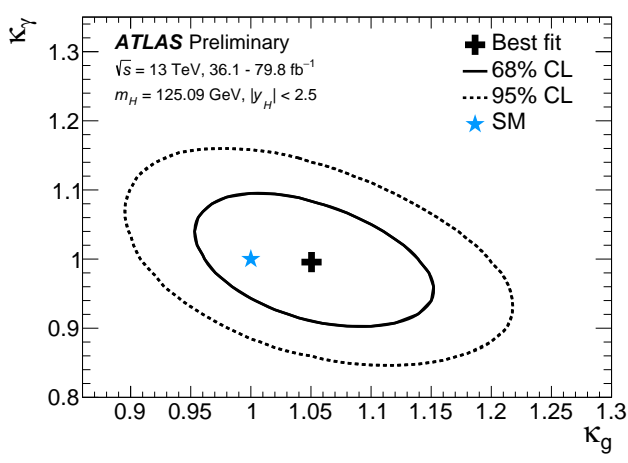

(a)

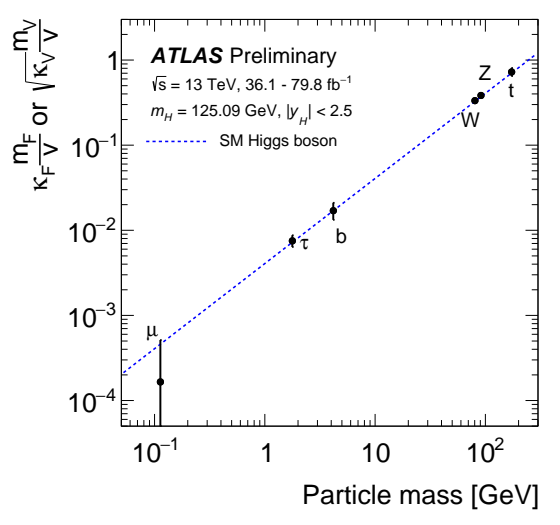

(b)

Figure 2: (a) Measured effective coupling strengths modifiers for gluons $\left(\kappa_{g}\right)$ and photons $\left(\kappa_{\gamma}\right)$ when probing non-SM contributions in the loops. (b) Reduced coupling strength modifiers $\kappa_{F} m_{F} / v$ for fermions $(F=t, b, \tau, \mu)$ and $\sqrt{\kappa_{V}} m_{V} / v$ for weak gauge bosons $(V=W, Z)$ as a function of their masses $m_{F}$ and $m_{V}$, when assuming the SM structure of loop processes.

\section{Conclusions}

The results of many Higgs boson analyses, performed by the ATLAS collaboration using up to $80 \mathrm{fb}^{-1}$ of Run- 2 collision data, have been combined. The differential distributions predicted by state-of-the-art SM calculations have been found in good agreement with the measurements of the $H \rightarrow \gamma \gamma$ and $H \rightarrow Z Z^{*} \rightarrow 4 \ell$ channels. The global combination of seven analyses achieves precise measurements of the Higgs boson production modes and delivers a broad set of results on the Higgs couplings. All measurements show an excellent compatibility with the SM predictions. Stringent limits are set when the results are interpreted in terms of the benchmark 2HDM or hMSSM models.

\section{References}

[1] ATLAS Collaboration, Phys. Rev. D 98 (2018) 052005 [hep-ex: 1802.04146 ]

[2] ATLAS Collaboration, JHEP 10 (2017) 132 [hep-ex:1708.02810]

[3] ATLAS Collaboration, Phys. Lett. B 786 (2018) 114 [hep-ex: 1805.10197$]$

[4] ATLAS Collaboration, ATLAS-CONF-2018-031

[5] ATLAS Collaboration, ATLAS-CONF-2018-028

[6] ATLAS Collaboration, ATLAS-CONF-2018-018

[7] ATLAS Collaboration, ATLAS-CONF-2018-004

[8] ATLAS Collaboration, ATLAS-CONF-2018-021

[9] ATLAS Collaboration, JHEP 12 (2017) 024 [hep-ex: 1708 . 03299]

[10] ATLAS Collaboration, ATLAS-CONF-2018-026

[11] ATLAS Collaboration, Phys. Lett. B 784 (2018) 173 [hep-ex: 1806.00425$]$ 